\title{
Testing the Kerr Metric with X-Ray Reflection Spectroscopy of Mrk 335 Suzaku Data
}

\author{
Kishalay Choudhury ${ }^{1}$, Sourabh Nampalliwar ${ }^{2}$, Askar B. Abdikamalov ${ }^{1}$, Dimitry Ayzenberg ${ }^{1}$, Cosimo Bambi ${ }^{1,2,5}$ (D), \\ Thomas Dauser ${ }^{3}$, and Javier A. García $4,3,5$ \\ ${ }^{1}$ Center for Field Theory and Particle Physics and Department of Physics, Fudan University, 200438 Shanghai, People's Republic of China \\ ${ }^{2}$ Theoretical Astrophysics, Eberhard-Karls Universität Tübingen, D-72076 Tübingen, Germany; bambi@ fudan.edu.cn \\ ${ }^{3}$ Remeis Observatory \& ECAP, Universität Erlangen-Nürnberg, D-96049 Bamberg, Germany \\ ${ }^{4}$ Cahill Center for Astronomy and Astrophysics, California Institute of Technology, Pasadena, CA 91125, USA \\ Received 2018 September 20; revised 2019 April 24; accepted 2019 May 25; published 2019 July 9
}

\begin{abstract}
Einstein's gravity has undergone extensive tests in the weak field gravitational limit, with results in agreement with theoretical predictions. There exist theories beyond general relativity (GR) which modify gravity in the strong field regime but agree with GR in the weak field. Astrophysical black holes are believed to be described by the Kerr metric and serve as suitable candidates to test strong gravity with electromagnetic radiation. We perform such a test by fitting one Suzaku data set of the narrow-line Seyfert 1 (NLS1) galaxy Mrk 335 with X-ray reflection spectroscopy, using the Johannsen metric to model the black hole spacetime and test for deviations from Kerr. We find the data is best modeled with a hybrid model that includes both partial covering absorption and a reflection component. This is the first time such a model has been proposed for a high-flux (low reflection) Mrk 335 data set. We constrain the Johannsen deformation parameter $\alpha_{13}$ to $-1.5<\alpha_{13}<0.6$ with spin parameter $a_{*}>0.8$, and the $\alpha_{22}$ parameter to $-0.4<\alpha_{22}<2.1$ with $a_{*}>0.7$, both at the $99 \%$ confidence level. Although additional solutions at large deviations from the Kerr metric show statistical similarity with the ones above, further analysis suggests these solutions may be manifestations of uncertainties beyond our control and do not represent the data. Hence, our results are in agreement with the idea that the supermassive compact object at the center of Mrk 335 is described by the Kerr metric.
\end{abstract}

Key words: black hole physics - galaxies: active - methods: data analysis - stars: black holes - techniques: imaging spectroscopy - X-rays: individual (Mrk 335)

\section{Introduction}

Over a hundred years old, Albert Einstein's theory of general relativity has been tested with observations since it was proposed and agreement has been found in numerous cases spanning across a multitude of domains, notably in weak field experiments of the solar system and for radio observations of binary pulsars (Will 2014). Testing gravity in the strong field regime has gained popularity among both the electromagnetic (EM) radiation and the gravitational wave community, with astrophysical black holes proving to be the perfect candidates for carrying out such tests (Yunes \& Siemens 2013; Bambi \& Nampalliwar 2016; Johannsen 2016; Yagi \& Stein 2016; Bambi 2017a; Bambi et al. 2017).

In four-dimensional Einstein gravity, the Kerr metric is the only vacuum black hole solution of the field equations which is regular on and outside the event horizon, under standard assumptions like stationarity and asymptotic flatness, a consequence of the no-hair theorem (Kerr 1963; Carter 1971; Robinson 1975). The general consensus is that the Kerr metric describes the spacetime around astrophysical black holes (Price 1972; Bambi et al. 2009, 2014). But a number of alternative theories predict macroscopic deviations from the Kerr spacetime (Mathur 2005; Dvali \& Gomez 2013; Giddings 2014; Berti et al. 2015; Giddings 2017). This makes it imperative to conduct tests on the Kerr hypothesis. Observations of the X-ray reflection spectrum from the black hole neighborhood are particularly interesting, as they can be used to test strong gravity by studying radiation emitted from regions very close to the black hole (Fabian et al. 2000). This

\footnotetext{
5 Alexander von Humboldt Fellow.
}

method has been developed over the years by assuming the Kerr metric describes the spacetime around the central compact object, in both active galactic nuclei (AGNs) and black hole binaries (BHB). Blurred and distorted emission features can be seen around the reflecting regions of the accretion disk due to relativistic effects, leading to measurements of inner disk radius and black hole spin (Fabian et al. 1989; Laor 1991; Brenneman \& Reynolds 2006; Reynolds \& Fabian 2008). The most notable and interesting feature is the $\mathrm{Fe}-\mathrm{K} \alpha$ emission complex (e.g., Guilbert \& Rees 1988; Lightman \& White 1988; Fabian et al. 1989; George \& Fabian 1991). The utilization of X-ray reflection spectroscopy for strong gravity tests has been examined in the last decade (Schee \& Stuchlík 2009; Bambi 2013; Johannsen \& Psaltis 2013; Jiang et al. 2015a, 2015b; Bambi et al. 2016; Zhou et al. 2016; Ni et al. 2016; Nampalliwar et al. 2018).

The X-ray blurring code RELXILL is currently the most popular relativistic reflection model in use that describes the reflection spectrum of optically thick, geometrically thin accretion disks (Novikov \& Thorne 1973) around black holes (García et al. 2014). The code is the combination of the convoluted (RELCONV) flavor of the RELLINE relativistic smearing model (Dauser et al. 2010, 2013) and the emissionangle-dependent, non-relativistic, local disk reflection code XILLVER (García \& Kallman 2010; García et al. 2013). RELCONV was recently modified by some of us to allow for the use of a non-Kerr metric for the purpose of testing gravity (Bambi et al. 2017). Our relativistic blurring code RELXILL_NK has been designed to incorporate any well-behaved, stationary, axisymmetric, and asymptotically flat spacetimes, including parameterized metrics that deform Kerr and solutions in modified gravity theories. Recent results obtained with the 
code for quantifying possible Kerr deviations with X-ray data of multiple sources have been summarized in Bambi et al. (2018). The first non-Kerr metric implemented in RELXILL_NK was the axisymmetric metric proposed in Johannsen (2013). Additional metrics implemented in the framework include the conformal metric of Bambi et al. (2017; see Zhou et al. 2018) and the axisymmetric metric in Konoplya et al. (2016; see Nampalliwar et al. 2019).

The paper is structured as follows. We review the Johannsen metric (expressed in the convention assuming the natural units $G_{\mathrm{N}}=c=1$ ) in Section 2 for the relativistic blurring code used. Our source is presented in Section 3.1, with the choice of the data set described in Section 3.2. We explain the data reduction methodology in Section 3.3, the listing and briefly explain the model components used for the data analysis in Section 3.4. A justification of the components and key observations leading to our presented results in Section 4 are discussed in Section 5. Finally, we express our concluding remarks and mention possible shortcomings in Section 6.

\section{RELXILL_NK: The Metric}

One parametrically deformed metric to test the Kerr hypothesis is the Johannsen metric (Johannsen 2013) that we implement in our code RELXILL_NK. Note that the metric is neither a solution of Einstein's field equations nor of any wellmotivated modified theory of gravity. We can, however, consider it as a phenomenological hypothesis and conduct strong field tests of the no-hair theorem in general classes of gravity theories.

The line element of the Johannsen metric in Boyer-Lindquist coordinates, with the convention $(-+++)$, is given as

$$
\begin{aligned}
d s^{2}= & -\frac{\tilde{\Sigma}\left(\Delta-a^{2} A_{2}^{2} \sin ^{2} \theta\right)}{\left[\left(r^{2}+a^{2}\right) A_{1}-a^{2} A_{2} \sin ^{2} \theta\right]^{2}} d t^{2} \\
& -\frac{2 a\left[\left(r^{2}+a^{2}\right) A_{1} A_{2}-\Delta\right] \tilde{\Sigma} \sin ^{2} \theta}{\left[\left(r^{2}+a^{2}\right) A_{1}-a^{2} A_{2} \sin ^{2} \theta\right]^{2}} d t d \phi \\
& +\frac{\tilde{\Sigma}}{\Delta A_{5}} d r^{2}+\tilde{\Sigma} d \theta^{2} \\
& +\frac{\left[\left(r^{2}+a^{2}\right)^{2} A_{1}^{2}-a^{2} \Delta \sin ^{2} \theta\right] \tilde{\Sigma} \sin ^{2} \theta}{\left[\left(r^{2}+a^{2}\right) A_{1}-a^{2} A_{2} \sin ^{2} \theta\right]^{2}} d \phi^{2},
\end{aligned}
$$

where

$$
\begin{aligned}
A_{1}(r) & =1+\sum_{n=3}^{\infty} \alpha_{1 n}\left(\frac{M}{r}\right)^{n} \\
A_{2}(r) & =1+\sum_{n=2}^{\infty} \alpha_{2 n}\left(\frac{M}{r}\right)^{n} \\
A_{5}(r) & =1+\sum_{n=2}^{\infty} \alpha_{5 n}\left(\frac{M}{r}\right)^{n} \\
\Delta & \equiv r^{2}-2 M r+a^{2} \\
\tilde{\Sigma} & =r^{2}+a^{2} \cos ^{2} \theta+f(r) \\
f(r) & =\sum_{n=3}^{\infty} \epsilon_{n}\left(\frac{M^{n}}{r^{n-2}}\right)
\end{aligned}
$$

with mass $M$ and spin parameter $a=J / M$ of the black hole, where $J$ is the spin angular momentum of the black hole. When the deviation functions $A_{1}=A_{2}=A_{5}=1$ and $f(r)=0$, Equation (1) reduces to the Kerr metric. We test for one deformation parameter at a time, keeping all others identically zero. Thus, we present two sets of results, one each for $\alpha_{13}$ and $\alpha_{22}$. We pick $\alpha_{13}$ and $\alpha_{22}$ because they are expected to have the strongest impact on the reflection spectrum (Bambi et al. 2017), thus their constraints will be the strongest.

Note that there exists a degeneracy between deformation parameters (and among deformation parameters and other model parameters) in terms of their effect on the relativistic blurring, and a systematic study of various kinds of degeneracies is currently underway. But allowing for multiple simultaneous deformation parameters in our reflection model is currently computationally unfeasible owing to memory issues with the analysis package and huge FITS files requirements.

In order to avoid pathologies in the spacetime, the following limits on $\alpha_{13}$ and $\alpha_{22}$ are imposed (Johannsen 2013; Bambi et al. 2017):

$$
\alpha_{13}>-\frac{1}{2}\left(1+\sqrt{1-a_{*}^{2}}\right)^{4}
$$

$$
-\left(1+\sqrt{1-a_{*}^{2}}\right)^{2}<\alpha_{22}<\frac{\left(1+\sqrt{1-a_{*}^{2}}\right)^{4}}{a_{*}^{2}},
$$

where $a_{*}=a / M$ is the dimensionless spin parameter present in our code.

\section{Data Analysis}

\subsection{The Source}

The narrow-line Seyfert 1 (NLS1) AGN Mrk 335 $(z=0.0258)$ has been found to host a supermassive black hole at its center with reverberation-mapped mass $M_{\bullet} \approx$ $2.6 \times 10^{7} M_{\odot}$ (Grier et al. 2012). First detected in X-rays by UHURU (Tananbaum et al. 1978), Mrk 335 has been observed and studied numerous times by various X-ray observatories such as ASCA, Swift, Suzaku, XMM-Newton, and NuSTAR (e.g., Ballantyne et al. 2001; Gondoin et al. 2002; Crummy et al. 2006; Grupe et al. 2007, 2008, 2012; Longinotti et al. 2007; O'Neill et al. 2007; Larsson et al. 2008; Patrick et al. 2011; Gallo et al. 2013, 2015, 2019; Walton et al. 2013; Longinotti et al. 2013; Parker et al. 2014; Wilkins \& Gallo 2015; Keek \& Ballantyne 2016; Ballantyne 2017; Beheshtipour et al. 2017). It is an extremely variable source, exhibiting more than a factor of 10 fluctuation in the X-ray flux over the past $15 \mathrm{yr}$. The source has been confirmed to have a Compton reflection component, and a strong soft excess and $\mathrm{Fe}-\mathrm{K} \alpha$ line broadening. Distinguishing Mrk 335 data at low energies $(<10 \mathrm{keV})$ between partial absorption and relativistic reflection was a challenge until the first pressing evidence for relativistic reflection from the accretion disk in the AGN was presented by Kara et al. (2013) by studying X-ray reverberation time lags. AGNs exhibit the highest complexity in low-flux states.

\subsection{Data}

Table 1 presents a list of Mrk 335 data available online for the Suzaku, XMM-Newton, and NuSTAR missions. Mrk 335 was observed in a high-flux state on 2006 June 21 (Obs. ID: 701031010) with Suzaku (Mitsuda et al. 2007) for an X-ray Imaging Spectrometer (XIS, Koyama et al. 2007) net exposure time of $151 \mathrm{ks}$. It is this data set that we will analyze in the present work owing to its limited complexity, good photon counting statistics, and the observation of relativistic reflection 
Table 1

Mrk 335 Archived X-Ray Data Studied in the Literature for Select Missions

\begin{tabular}{lccc}
\hline \hline Instrument & Obs. ID & $\begin{array}{c}\text { Date } \\
\text { [yyyy/mm/dd] }\end{array}$ & $\begin{array}{c}\text { Exposure Time } \\
(\mathrm{ks})\end{array}$ \\
\hline Suzaku & 701031010 & 2006 Jun 21 & 151.3 \\
& 708016010 & 2013 Jun 11 & 144.0 \\
& 708016020 & 2013 Jun 14 & 154.8 \\
\hline XMM-Newton & 0101040101 & 2000 Dec 25 & 36.9 \\
& 0101040701 & 2000 Dec 25 & 10.9 \\
& 0306870101 & 2006 Jan 3 & 133.2 \\
& 0510010701 & 2007 Jul 10 & 22.6 \\
& 0600540601 & 2009 Jun 11 & 132.3 \\
& 0600540501 & 2009 Jun 13 & 82.6 \\
& 0741280201 & 2015 Dec 30 & 140.4 \\
\hline NuSTAR & 60001041002 & 2013 Jun 13 & $21.3 / 21.5$ \\
(FPMA/B) & 60001041003 & 2013 Jun 13 & $21.5 / 21.3$ \\
& 60001041005 & 2013 Jun 25 & $93.0 / 92.9$ \\
& 80001020002 & 2014 Sep 20 & $68.9 / 68.8$ \\
\hline
\end{tabular}

in it. The chosen observation uses Walton et al. (2013) as a basis, which is a survey paper investigating relativistic disk reflection for 25 "bare" type 1 AGN Suzaku data with little or no complicated intrinsic absorption. The cited work presented findings using the chosen Mrk 335 data set with blurred reflection and showed the presence of relativistically skewed $\mathrm{Fe}$ line and a Compton turnover at $E>10 \mathrm{keV}$.

We compare other data in Table 1 against our choice here. Each individual front-illuminated (FI) XIS spectrum (Section 3.3) in our high-flux observation has an effective exposure time ( $\sim 133 \mathrm{ks})$ similar to the total exposure of the XMM-Newton high-flux 2006 data set instead, giving us better counts with Suzaku. An added detector advantage of Suzaku XIS is that it had relatively lower intrinsic background contamination compared to EPIC cameras (Jansen et al. 2001), by construction. Gallo et al. (2013), with a combined $\sim 200 \mathrm{ks} 2009$ XMM-Newton exposure time, show weak constraints on $a_{*}$ (similar to Walton et al. 2013) with a deeper analysis of the intermediate-flux data. The remaining data sets (all in low-flux state) have much lower effective exposures and net counts for both satellite missions, in addition to significant warm absorber modification (likely to heighten intercomponent degeneracies) of the reflection data. Flaring in the $\sim 140 \mathrm{ks} 2015$ $X M M-N e w t o n$ low-flux observations results in a $\sim 20$ ks cutdown, and the remaining observation is photon-starved (Gallo et al. 2019).

It is essential to state that there exist simultaneous highenergy exposures of Mrk 335 with NUSTAR (Harrison et al. 2013), which encompasses the full reflection band when used with low-energy satellite data. The only simultaneous observations available as of 2019 April (Table 1) are with the low-flux 2013 SUZAKU exposures. Although these Suzaku observations amount to $\sim 300 \mathrm{ks}$ net exposure over $\sim 7.7$ days, there is a threefold issue that builds up problems using these observations. (1) The observations are photon-starved with $<8 \times 10^{4}$ FI XIS counts (Gallo et al. 2015). (2) The NuSTAR coverage is short, corresponds to a very small part of the wide Suzaku exposures, and has incredibly low statistics (Parker et al. 2014). (3) $\mathrm{A} \sim 90-100 \mathrm{ks}$ flare $\sim 250 \mathrm{ks}$ in the Suzaku observations softens the source spectrum (Wilkins \& Gallo 2015), thereby further reducing good intervals for a simultaneous low-flux study, especially creating problems with time-averaged analysis. A simultaneous analysis of Mrk 335 with NUSTAR, to date, is not feasible to study strong gravity with reflection spectroscopy owing to a lack of decent coverage.

The struggle with poor detection at the low fluxes with this highly variable source is likely to make it difficult to constrain sensitive parameters such as the deformation parameters. The aim of this work is to study the constraints on possible deviations away from the Kerr metric using data quality with little complexity to avoid complicated intercomponent degeneracies that may shadow our findings. In general, inclusion of other observations may provide more data. But this does not necessarily imply better constraints, since at some point systematic uncertainties dominate over statistical uncertainties (see e.g., Tripathi et al. 2019a; Xu et al. 2018; Zhang et al. 2019).

\subsection{Reduction}

HEASOFT v6.25 reduction and analysis package was used to process unfiltered event files of the XIS CCDs following the Suzaku Data Reduction Guide, ${ }^{6}$ using XIS CALDB v20160607. Task aepipeline was run to create cleaned event files for the FI CCDs XIS0, XIS2 (non-operational since 2006 November), and XIS3. Back-illuminated (BI) CCD XIS1 was not used because its sensitivity is relatively low at $\mathrm{Fe} \mathrm{K}$ energies. Source (on-center) and background regions of 3.5 arcmin radii were extracted for each FI CCD on the SAOImage DS 9 imaging and data visualization application. ${ }^{7}$ Backgrounds were selected from the same CCD by avoiding the source and the ${ }^{55} \mathrm{Fe}$ calibration sources at the corners of the CCD. Unbinned source and background spectra for each CCD were extracted using the XSELECT tool, ensuring the cutoff rigidity was set $>6 \mathrm{GeV}$ (Walton et al. 2013) to account for proper non-X-ray background subtraction. The redistribution matrix file (RMF) and the ancillary response file were created using the tools XISRMFGEN and XISARFGEN, ${ }^{8}$ respectively. Since we are interested in average spectral properties, the FI CCD spectra and responses were co-added using the FTOOL ADDASCASPEC. The spectra and response files were physically rebinned using the tools RBNPHA and RBNRMF, respectively, with the variable binning scheme shown in the aforementioned guide. On top of this, to reduce bias imposed by a minimum grouping technique (e.g., see Figure 7 in Choudhury et al. 2017) and ensure high signal-to-noise ratio, we grouped our time-averaged XIS data set to $100 \mathrm{cts}^{\mathrm{bin}}{ }^{-1}$ using the FTOOL GRPPHA. Apart from data below $0.6 \mathrm{keV}$ and above $10 \mathrm{keV}$, that between 1.7 and $2.5 \mathrm{keV}$ were also ignored due to uncertainties in detector calibration around $\mathrm{Si} \mathrm{K}$ edge. The resulting data set had $\approx 5 \times 10^{5}$ total photon counts between 0.6 and $10 \mathrm{keV}$ with a net count rate of $1.222 \pm 0.002 \mathrm{~s}^{-1}$ and a very low background contamination (1.2\%).

HXD/PIN (Takahashi et al. 2007) data was also reduced similarly: employing aepipeline and then the FTOOL HXDPINXBPI using latest CALDB v20110913. Evidence of a Compton hump was seen, with a turnover $\sim 20 \mathrm{keV}$. However, owing to poor statistics $(\sim 6 \%$ of total counts between $0.6-25 \mathrm{keV}$ in PIN) and high errors on the data, in addition to no significant contribution to the analysis of the

\footnotetext{
6 https://heasarc.gsfc.nasa.gov/docs/suzaku/analysis/abc/

7 http://ds9.si.edu/site/Home.html

8 ftp://legacy.gsfc.nasa.gov/suzaku/doc/xrt/suzakumemo-2011-01.pdf
} 


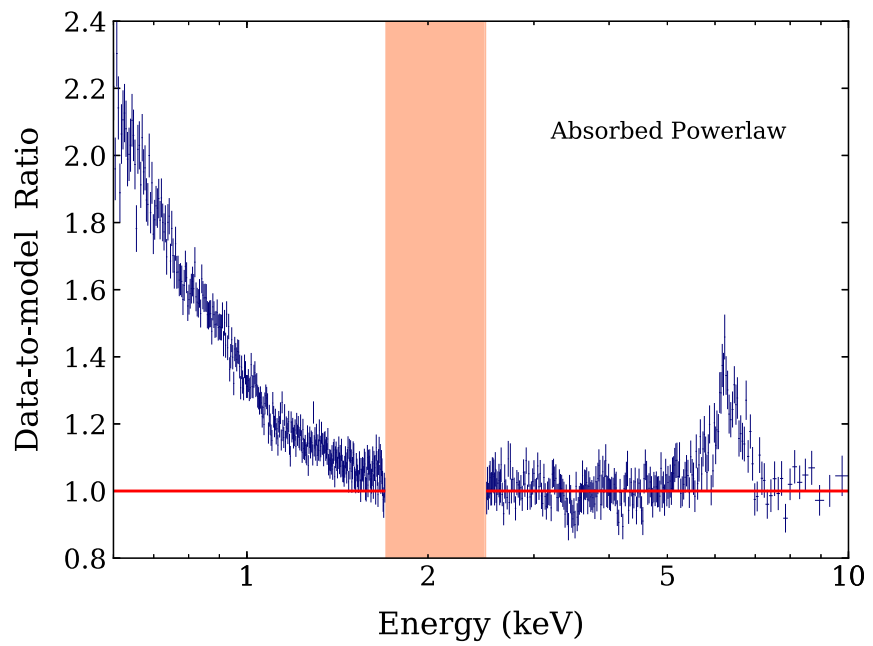

Figure 1. Phenomenological power-law fit to the time-averaged data with $\Gamma \simeq 2.09$. The shaded energy range $(1.7-2.5 \mathrm{keV})$ was not included due to calibration issues. The spectrum was fit from $2.5-4.0$ and $8-10 \mathrm{keV}$, and then plotted after introducing the $0.6-1.7$ and $4-8 \mathrm{keV}$ bands, to bring out the $\mathrm{Fe}$ and soft band features. The plot was rebinned on XSPEC for clarity.

reflection spectrum, the PIN data set was not included for our test of gravity.

\subsection{Modeling}

For our work in this paper we made use of the X-ray spectral fitting package XSPEC V12.10.1b (Arnaud 1996). The latest (as of 2017 September 4) ionization balance calculation (v3.0.7) database was imported additionally to be able to properly account for up-to-date modeling of X-ray emission and absorption from complex spectra. ${ }^{9}$

To bring out features in our $0.6-10 \mathrm{keV}$ spectrum, we first try a phenomenological model — an absorbed power-law fit (Figure 1). The galactic H I column has been fixed to $3.56 \times 10^{20} \mathrm{~cm}^{-2}$ (Kalberla et al. 2005) with the ISM grain absorption model TBABS. The cross sections are set by the abund wilm command (Wilms et al. 2000). The data show clear signs of a broad Fe K region with a strong soft excess. We ran the fit and obtained poor statistics $\left(\chi^{2} /\right.$ d.o.f. $\left.=8011.66 / 971\right)$. The $2-10 \mathrm{keV}$ absorbed power-law flux is $\simeq 1.39 \times 10^{-11} \mathrm{erg} \mathrm{cm}^{-2} \mathrm{~s}^{-1}$. Following what we see, we tested out several model combinations on the XIS data set. But we only display results from the physically and statistically relevant models, and explain in Section 5 why others were not favored.

For every combination we start with the possibility that only one deformation $\left(\alpha_{13}\right.$ or $\left.\alpha_{22}\right)$ in the Johannsen metric (See Equation (1)) is non-zero, leaving it as a fit parameter and assuming all other deformation parameters are equal to 0. For the sake of avoiding unphysically extreme inner disk inclination fits to a Seyfert 1 AGN (e.g., Nandra et al. 1997; Rakshit et al. 2017), we limit the upper hard limit of the inclination parameter in RELXILL_NK to $75^{\circ}$ instead. Tables 2 and 3 display the best-fit parameters obtained for all models with $\alpha_{13}$ $\neq 0$ and $\alpha_{22} \neq 0$, respectively, while Figure 2 shows the unfolded spectrum with the contributions of the additive model components, for our best-fit model.

\footnotetext{
9 http://www.atomdb.org/download.php
}

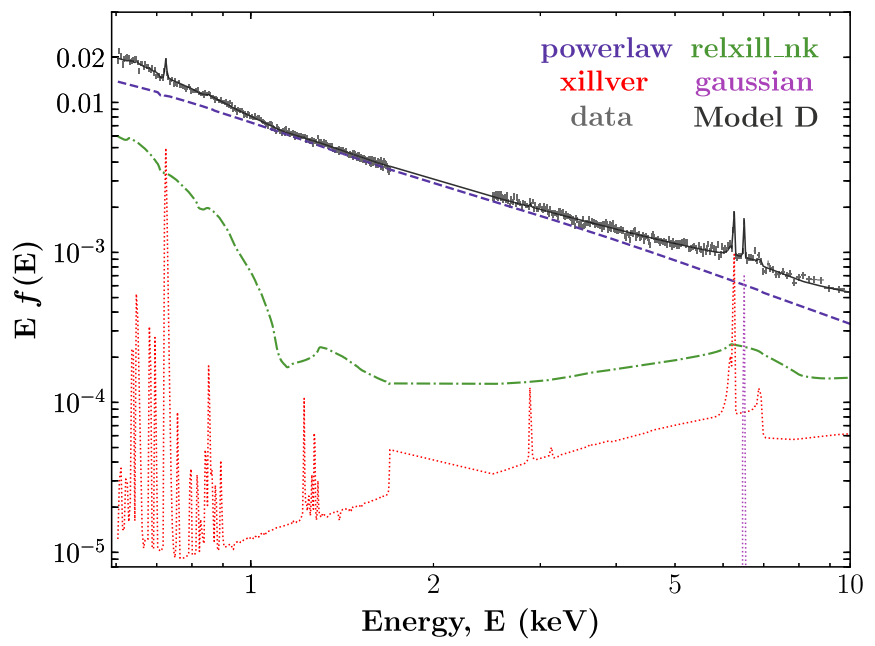

Figure 2. Unfolded spectrum with the best-fit model D. The vertical axis is in units of photons $\mathrm{cm}^{-2} \mathrm{~s}^{-1}$. Contributions from different model components are shown in different colors.

\subsubsection{Model A}

TBABS * (ZPOWERLW + RELXILL_NK)

We added our non-Kerr blurred reflection model RELXILL_NK here with the reflection fraction $\left(R_{f}\right)$ parameter fixed at -1 , corresponding to a pure reflection signal. The redshifted power-law component (PLC) takes into account the continuum signal separately, with the photon indices $\Gamma$ linked between the two. Since an $E<10 \mathrm{keV}$ data set is not suited for constraining the high-energy cutoff $\left(E_{\text {cut }}\right)$ in AGN (e.g., refer to Tortosa et al. 2018, for typical values), $E_{\text {cut }}$ was fixed at the default value of $300 \mathrm{keV}$ (Keek \& Ballantyne 2016). Model A $\chi^{2}$ plots in Figures 3(a) and (b) show the immense improvement in the fits, with $\Delta \chi^{2}>6800$. Most of the soft excess is accounted for by the blurred reflector (Miller 2007). This suggests the need for a reflection-dominated component (RDC). Still, the convergence is poor and suggests the necessity to consider narrow emission residuals (evident from the $\chi^{2}$ plots).

\subsubsection{Model B}

\section{TBABS * (ZPOWERLW + RELXILL_NK + XILLVER)}

We made use of the XILLVER reflection code here to account for narrow, non-relativistic reflected emission in the spectrum. From preliminary fits we find that leaving the ionization parameter free here leads to fits of $\log \xi_{\text {unblurred }} \approx 0$. Similarly, the fit is insensitive to the value of the inclination parameter of the distant reflector. Hence, we fixed ionization to 0 and inclination to the default. The iron abundance $A_{\mathrm{Fe}}$ was linked to that of the blurred reflector with the general idea that the cold reflector could well be part of the same galaxy. Leaving $A_{\mathrm{Fe}}$ fixed at solar value returns poor fits. On the other hand, leaving it free results in extremely high, unconstrained abundances at the upper bound. The addition is $>4 \sigma$ significant for $\alpha_{13}$ $\left(\Delta \chi^{2} \simeq 26\right)$ and $>5.5 \sigma$ significant for $\alpha_{22}\left(\Delta \chi^{2} \simeq 43\right)$, for 1 extra degree of freedom (d.o.f.). Model B $\chi^{2}$ plots have not been shown because they are very similar to Model C's. (See below.) The middle panel in Figures 3(a) and (b) clearly shows that the narrow Fe core was fit by the model. 
Table 2

Best-fit Parameter Values Obtained Employing Models A to D with Only Deformation Parameter $\alpha_{13} \neq 0$

\begin{tabular}{|c|c|c|c|c|c|}
\hline \multirow{2}{*}{ Component } & \multirow{2}{*}{ Parameter [Unit] } & \multicolumn{4}{|c|}{ Model Values $^{\mathrm{a}}$} \\
\hline & & A & B & $\mathrm{C}$ & $\mathrm{D}$ \\
\hline$\overline{\text { ZPCFABS }}$ & $n_{\mathrm{H}}\left[10^{22} \mathrm{~cm}^{-2}\right]$ & 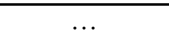 & $\ldots$ & 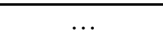 & $6.57_{-0.72}^{+0.88}$ \\
\hline \multirow{2}{*}{ ZPOWERLW } & PhoIndex & $2.35 \pm 0.01$ & $2.35 \pm 0.01$ & $2.35 \pm 0.01$ & $2.50_{-0.03}^{+0.02}$ \\
\hline & norm $_{1}\left[10^{-2} \mathrm{ph} \mathrm{cm}^{-2} \mathrm{~s}^{-1} \mathrm{keV}^{-1}\right]$ & $0.80 \pm 0.01$ & $0.80 \pm 0.01$ & $0.80 \pm 0.01$ & $1.09 \pm 0.06$ \\
\hline & $i\left[\operatorname{deg}\left({ }^{\circ}\right)\right]$ & $74.2_{-1.5}^{(P)}$ & $75.0_{-1.6}^{(P)}$ & $55.8_{-0.3}^{+0.9}$ & $67.4_{-3.9}^{+4.1}$ \\
\hline & $\log \xi$ & $1.23_{-0.11}^{+0.08}$ & $1.30_{-0.14}^{+0.01}$ & $1.25 \pm 0.05$ & $1.29_{-0.17}^{+0.03}$ \\
\hline & $A_{\mathrm{Fe}}$ & $2.30_{-0.34}^{+0.32}$ & $1.61_{-0.38}^{+0.44}$ & $1.39_{-0.40}^{+0.20}$ & $0.84_{-0.18}^{+0.23}$ \\
\hline & $\alpha_{13}$ & $-0.299_{-0.025}^{+0.006}$ & $-0.286_{-0.018}^{+0.025}$ & $-1.195_{-0.256}^{+0.121}$ & $0.214_{-1.103}^{+0.087}$ \\
\hline & norm $_{2}\left[10^{-4} \mathrm{ph} \mathrm{cm}^{-2} \mathrm{~s}^{-1} \mathrm{keV}^{-1}\right]$ & $3.54 \pm 0.17$ & $3.20_{-0.26}^{+0.20}$ & $2.74_{-0.10}^{+0.19}$ & $2.87_{-0.33}^{+0.22}$ \\
\hline & $\mathrm{EW}_{\mathrm{XXV}}[\mathrm{eV}]$ & $\cdots$ & $\cdots$ & $18.4_{-9.0}^{+9.1}$ & $28.5_{-9.4}^{+9.2}$ \\
\hline \multirow[t]{2}{*}{$\chi^{2} /$ d.o.f. } & $\ldots$ & $1155.28 / 964$ & $1128.78 / 963$ & $1119.02 / 961$ & $1024.99 / 959$ \\
\hline & $\cdots$ & $\approx 1.198$ & $\approx 1.172$ & $\approx 1.164$ & $\approx 1.069$ \\
\hline
\end{tabular}

Note. Errors are given for $90 \%$ confidence, unless explicitly stated. A single emissivity profile was adopted (Walton et al. 2013).

a " $(P)$ " against error values implies the parameter has no bound there.

Table 3

Best-fit Parameter Values Obtained Employing Models A to D with Only Deformation Parameter $\alpha_{22} \neq 0$

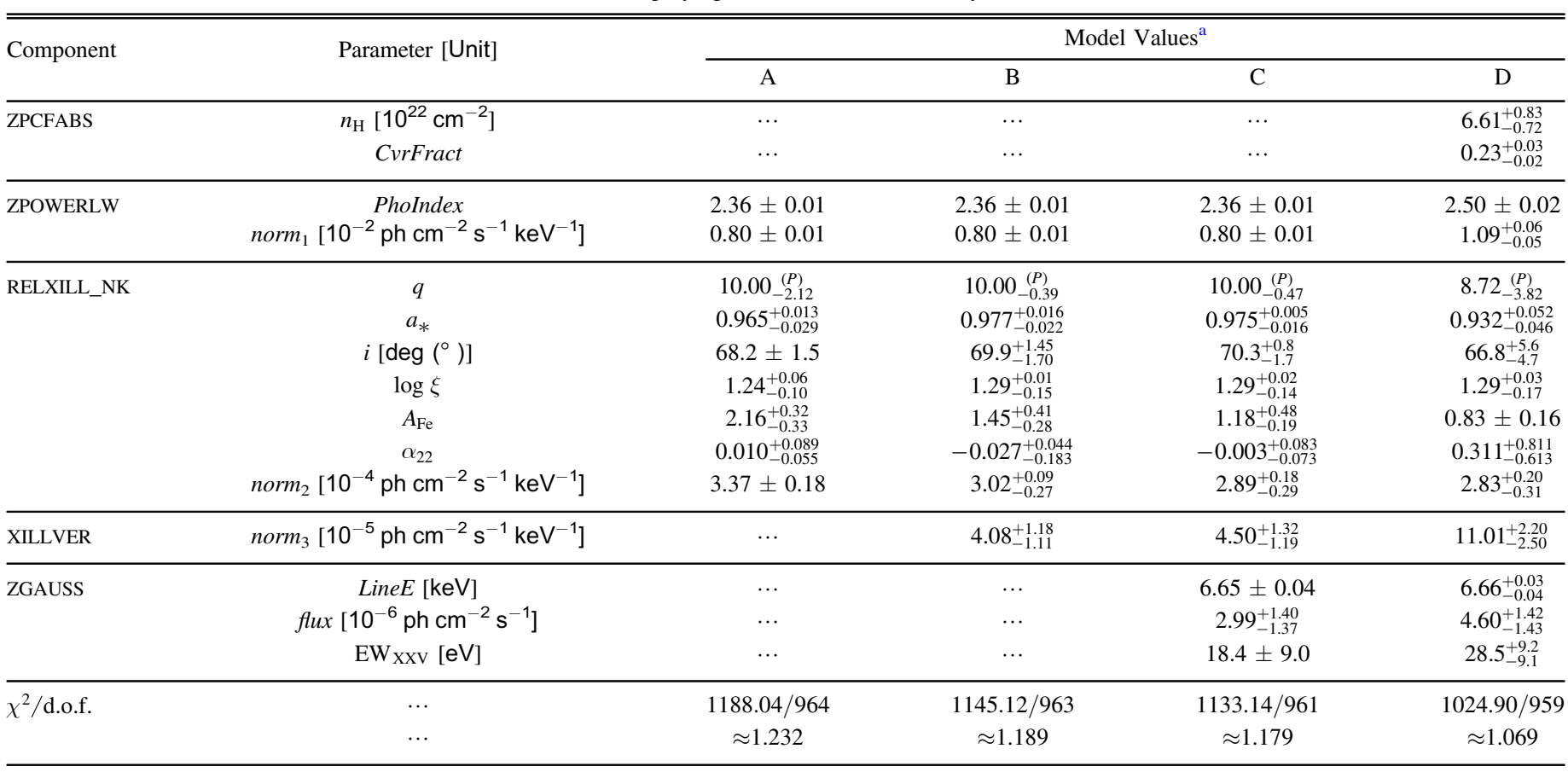

Note. Errors are given for $90 \%$ confidence, unless explicitly stated. A single emissivity profile was adopted (Walton et al. 2013).

a " $(P)$ " against error values implies the parameter has no bound there.

\subsubsection{Model C}

TBABS * (ZPOWERLW + RELXILL_NK + XILLVER + ZGAUSS)

We detected the presence of a narrow $(\sigma=10 \mathrm{eV}) \mathrm{Fe}-\mathrm{XXV}$ emission line at $E \simeq 6.65 \mathrm{keV}$ for both $\alpha_{13}$ and $\alpha_{22}$ cases.
Existence of this residual was also mentioned in Patrick et al. (2011) and Walton et al. (2013), who analyzed the same data set in Kerr spacetime. The improvement in the delta-fit statistic with the inclusion of such a line in the model agrees with Patrick et al. (2011) (Table 8 therein) at $\Delta \chi^{2} \simeq 10$. 


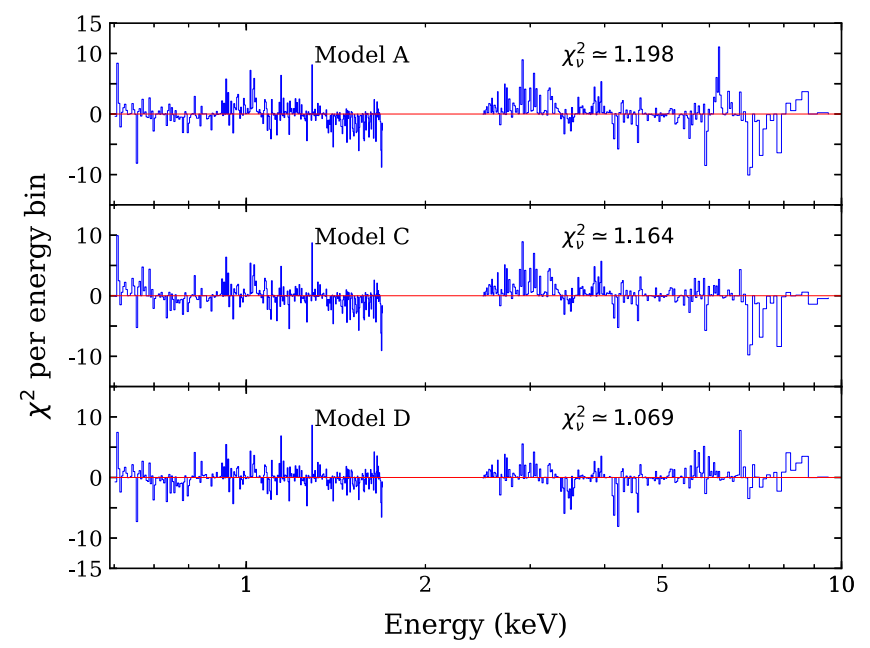

(a)

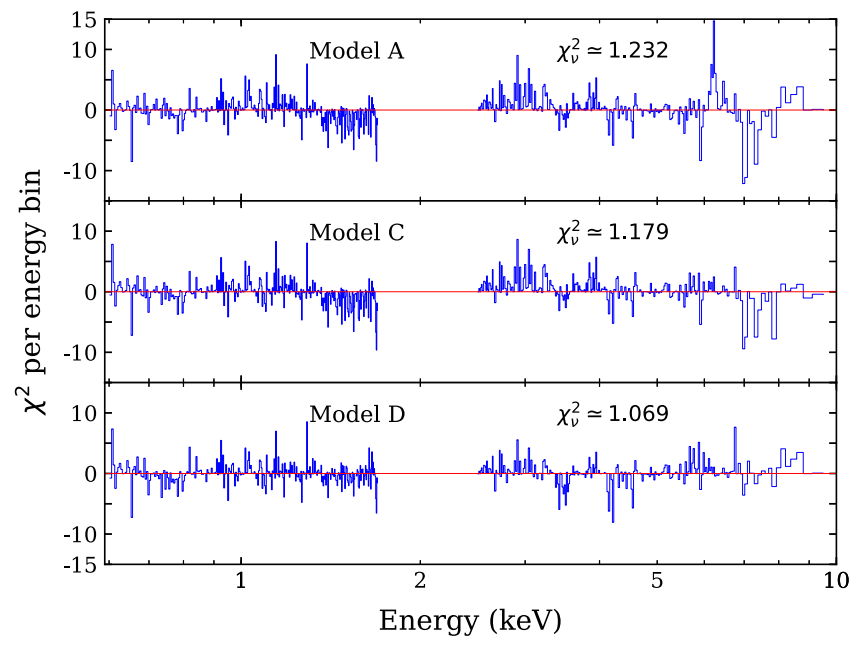

(b)

Figure 3. $\chi^{2}$ plots for models A, C, and D for $\alpha_{13} \neq 0$ (left) and $\alpha_{22} \neq 0$ (right) from Tables 2 and 3, respectively. Plots were rebinned on XSPEC. $\nu$ in $\chi_{\nu}^{2}$ represents the degrees of freedom (d.o.f.), where $\chi_{\nu}^{2}=\chi^{2} /$ d.o.f.

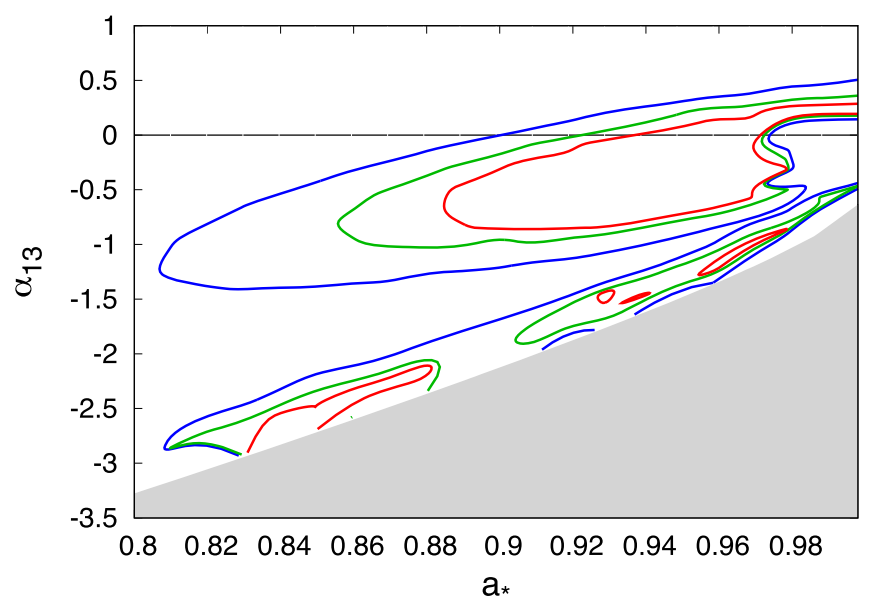

(a)

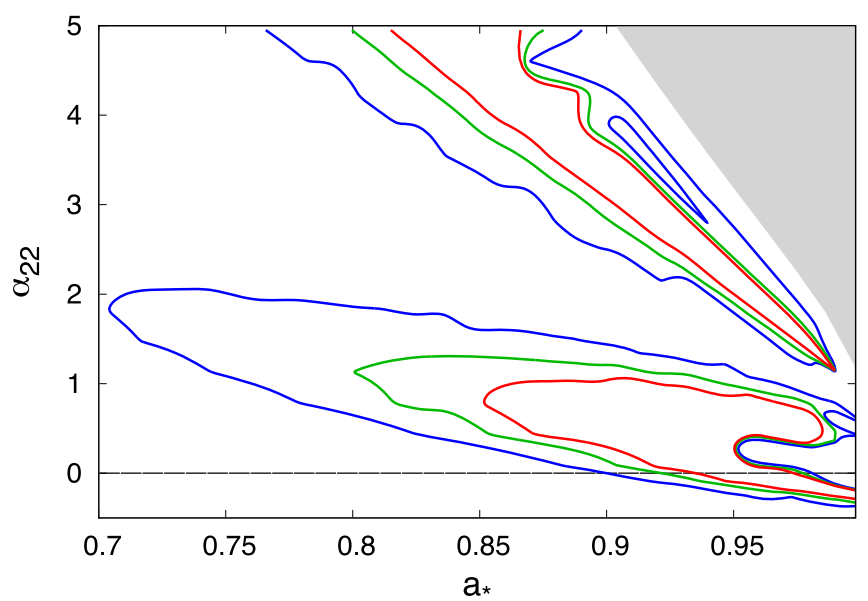

(b)

Figure 4. Two-dimensional degeneracy contours between the dimensionless spin parameter $a_{*}$ and Johannsen deformation parameters $\alpha_{13}$ (left) and $\alpha_{22}$ (right) for the best-fit model D. The dashed horizontal line at 0 marks the Kerr solution. The red, green, and blue contour lines indicate 68\%, $90 \%$, and $99 \%$ confidence levels, respectively. The shaded spaces in gray are forbidden regions within the metric that avoid some pathological properties as mentioned in Section 2.

\subsubsection{Model D}

\section{TBABS * [ZPCFABS * (ZPOWERLW + RELXILL_NK + XILLVER + ZGAUSS)]}

After Model C, we tried several alternative RDC combinations, briefly described in Section 5. But none of them seemed to improve the fit statistic. However, hints of absorption can be seen in the spectra. We turned to this possibility, and different absorbers and their combinations were examined. The simplest inclusion of one partial covering (ZPCFABS) proved satisfactory. The $\Delta \chi^{2}$ in the $\alpha_{13}$ and $\alpha_{22}$ cases were, respectively, 94 and 108 for 2 extra d.o.f. The parameters of the covering model are well constrained, as can be seen in Tables 2 and 3. The inclusion improved the fits with high significance $(>7 \sigma)$. The unfolded spectrum of model D is plotted in Figure 2, along with the contribution of each additive model component.

\section{Results}

The primary aim of this study is to provide constraints on deviations from the Kerr metric. Tables 2 and 3 list the values of $\alpha_{13}$ and $\alpha_{22}$ for each model. For the best-fit model, model D, the Kerr solution (corresponding to a deformation parameter equal to zero) is recovered in both cases. Because deformation parameters are strongly degenerate with the spin parameter, we show the two-dimensional spin-deformation contours in Figure 4 for $\alpha_{13}$ and $\alpha_{22}$, using the STEPPAR command on XSPEC. There is a clear degeneracy between the two parameters, which includes the Kerr solution in both cases. Apart from the usual contours observed in such studies (the near-Kerr contours; see Figure 2 in Tripathi et al. 2018 and Figure 2 in Xu et al. 2018), additional contours appear that exclude the Kerr solution at $3 \sigma$ (the far-Kerr contours). A thorough investigation (detailed in Appendix A) indicates that 
these far-Kerr contours are unreliable. We thus ascribe no significance to these. The constraints on the deformation parameters from these plots, after excluding the far-Kerr contours, are

$$
\begin{array}{lll}
\alpha_{13} \neq 0: & a_{*}>0.8 & -1.5<\alpha_{13}<0.6 \\
\alpha_{22} \neq 0: & a_{*}>0.7 & -0.4<\alpha_{22}<2.1 .
\end{array}
$$

We can make some comparisons for $\alpha_{13}$ with constraints from other studies. The constraints obtained here are better than those in Bambi et al. (2018; Figures 2 and 3 therein), which are updated versions of those in Cao et al. (2018) for both 2011 XMM-Newton data and simultaneous NuSTAR+Swift results of the NLS1 1H0707-495. The contours with Markov Chain Monte Carlo (MCMC) runs in Wang-Ji et al. (2018) for the popular X-ray BHB GX 339-4 with RXTE PCU-2 data are comparable to ours here. For both deformation parameters, results from Tripathi et al. (2018) on the NLS1 Ark 564 Suzaku XIS and from Xu et al. (2018) on the low-mass X-ray binary GS 1354-645 NuSTAR FPM analyses are stronger than ours here.

Note that one of the systematic effects that affects the uncertainty in deformation parameter values is the ISCO (innermost stable circular orbit) radius degeneracy between spin and the deformation parameter. (See, e.g., Figure 6 in Johannsen 2013 and Figure 12 in Tripathi et al. 2019b.) Lower spins result in weaker constraints on the deformation parameters. We can see this pattern in all the results. For GX 339-4, the spin is comparable to the spin here, and so is the $\alpha_{13}$ uncertainty; for Ark 564 and GS 1354-645, the spin is higher and is more strongly constrained, so $\alpha_{13}$ also has stronger constraints.

The spin recovered is consistent with the literature where Mrk 335 can be seen to have wide uncertainties on $a_{*}$ in the Kerr background at $90 \%$ confidence for one parameter of interest (e.g., $a_{*}>0.7$ in Gallo et al. 2013, with the $\sim 200 \mathrm{ks}$ XMM-Newton intermediate-flux data from 2009). Even Walton et al. (2013) presented a lower limit of 0.7 on $a_{*}$ with our data set. Our best-fit $a_{*}$ from Tables 2 and 3 are similar to those from Gallo et al. (2015), who use a broken emissivity profile instead. We roughly recover $0.93 \lesssim a_{*} \lesssim 0.97$ in the Kerr limit $\left(\alpha_{13}=\alpha_{22}=0\right)$ from both Figures 4(a) and (b), at $90 \%$ confidence.

\section{Discussion}

We now discuss some aspects of the models and the results.

\subsection{Soft Excess}

Absorption in the 1-3 keV band is evident from the absorbed power-law fit, with a strong soft excess $<1 \mathrm{keV}$, both being reported in Keek \& Ballantyne (2016). From model fits A to C we see tight parameter constraints, possibly indicating overestimation of error bars. Comparing with Patrick et al. (2011) and Walton et al. (2013), we observe a rise in $\Gamma$ and drop in $\xi$ with all four models. This line-continuum trade-off is possible with RELXILL (Choudhury et al. 2017). Recovering a relatively colder disk can also be responsible for causing the soft excess at lower energies, resulting from blending of multiple narrow emission lines that can be fit by blurred reflection (Miller 2007).

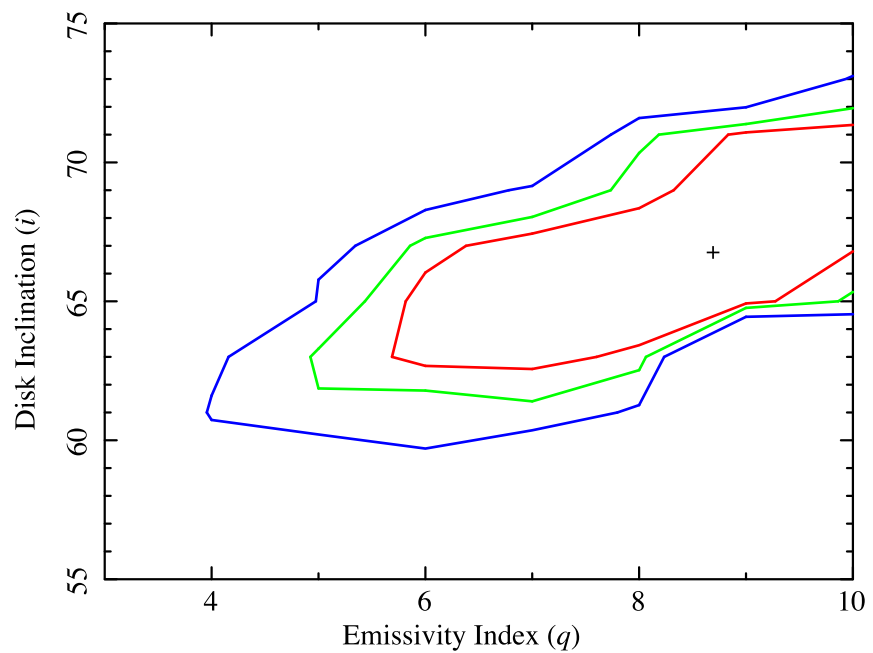

Figure 5. Contour plot between the inner disk inclination $i$ (in units of degrees) and emissivity index $q$ from model $\mathrm{D}, \alpha_{22} \neq 0$ case. The red, green, and blue contour lines indicate $68 \%, 90 \%$, and $99 \%$ confidence levels, respectively. The best fit is marked with a "+."

\subsection{Inclination Angle}

In general, a Seyfert 1 AGN (especially a NLS1) is seen to be more face-on than edge-on, while we obtain a best-fit $i \simeq$ $67^{\circ}$. This is comparatively higher than what Walton et al. (2013) report at similar reduced statistics, although we have higher precision. One possible explanation is as follows: the fit values in both Patrick et al. (2011) and Walton et al. (2013) seem to follow the $i-q$ degeneracy trend shown in Parker et al. (2014) (Figure 11 therein) for a time-averaged spectrum analyzed with RELXILL. We also find this degeneracy (see Figure 5) with a preference for $i>58^{\circ}$ at $99 \%$ confidence. Owing to limited spectral resolution, there could exist a preference for both parameters to yield high fit values in such case. Another possible explanation is that the broad-line region of the AGN is either absent or hidden from the observer, which makes us recover a higher angle (Giannuzzo \& Stirpe 1996).

\subsection{Absorption}

Although Model C here is analogous to the model in Walton et al. (2013, who employ REFLIONX instead; Ross \& Fabian 2005), we see that the fits are not satisfactory. We attempted to fit a doubly blurred reflection model (a second RELXILL_NK) with all parameters linked, except for the ionization and normalization (Cao et al. 2018; Tripathi et al. 2018), both with and without the distant reflector. We also inspected with multiple distant reflectors. The inclusions did not improve the fits, implying there may be no accretion disk inhomogeneities as such. A general (or multi-component) PLC + RDC model combination cannot account for this data set with RELXILL_NK. The spectra still had significant residuals beyond $2 \sigma$ significance at energies $<3 \mathrm{keV}$, which contains $\sim 80 \%$ of the total XIS counts.

To obtain a better fit, we looked at the $\chi^{2}$ plots. (See model $\mathrm{C}$ in Figures 3(a) and (b).) Absorption within the observed (redshifted source) frame $E \sim 7-8 \mathrm{keV}$ can be seen. Local absorption at the source frame seems possible, as mentioned in Longinotti et al. (2013) and Keek \& Ballantyne (2016; see Larsson et al. 2008 and Patrick et al. 2011 for contrary opinions). We decided to add absorbers to the model and found 
a better fit. While both ionized and un-ionized absorbers provided better fits, eventually the un-ionized absorber model ZPCFABS was found to be satisfactory. (See Appendix B for details.)

We now discuss the implications of the introduction of such an absorption model in the analysis. Even in the Kerr case, there are results in the literature where we see that a mild continuum absorption of the reflection data improves the fits. Very recent work on the NLS1 Mrk 766 shows that this hybrid scenario is possible and yields more reasonable estimates on the X-ray continuum absorption (Buisson et al. 2018). The referred work finds parameter constraints in line with ours after introducing a partial covering to their reflection model. One of the effects of such absorption is the increase in uncertainty on some or all parameters of interest. We can see such an effect on the estimation of the deformation parameters between Model D and all other models (see, Tables 2 and 3 ).

Model D, which includes the absorber, gives us a converging best-fit statistic $(\sim 1.07)$ that is similar to the best-fit model in Walton et al. (2013). Moreover, in Model D both the Fe-XXV line energy and the equivalent width $\left(\mathrm{EW}_{\mathrm{XXV}}\right)$ of the line are similar to that reported in Walton et al. (2013), after possible broadening introduced due to the adjustment offered by the partial covering, reducing the need for narrow emission lines. Additionally, we see in Model D an enhanced signal (rise in the intensity of the distant reflector from $\sim 12 \%$ in Model $\mathrm{C}$ to $\sim 28 \%$ in Model D compared to the total RDC contribution; a rise in $\Gamma$ by $\sim 6 \%$ ) as compensation for the flux absorbed, possibly from both the continuum and the cold reflector. At high $i \simeq 67^{\circ}$, the partial covering barely affects the net model complexity with a Compton-thin continuum absorption under a low $\sim 25 \%$ covering, pointing at possible line-of-sight obstruction by local (cold) matter. The net X-ray flux absorbed at the source rest frame from Model C to D is also low $\left(\Delta L_{0.6-10 \mathrm{keV}} \simeq(1-1.5) \times 10^{-2} \mathrm{erg} \mathrm{s}^{-1}\right)$.

\subsection{Caveats}

Our constraints from this work establish that the Kerr metric in Einstein's gravity is recovered at $99 \%$ confidence with the data analyzed. However, care needs to be taken while interpreting the results, as there are simplifications involved. The caveats can be divided into four categories:

1. There could exist intercomponent degeneracies, as we include multiple components, and this may add to the wide uncertainties seen in our results. Exempli gratia this is the first time that a partial covering of the reflection spectrum has been proposed for this source in a high-flux data set. Generically, partial absorption and relativistic emission are degenerate with respect to the spectral shape, and a more sophisticated analysis like MCMC is required to check for the robustness of such a combination.

2. The assumption that the intensity profile is a power law is also an approximation. Employing REFLIONX, Gallo et al. (2015) and Wilkins \& Gallo (2015) showed that the Mrk 335 corona during the Suzaku 2006 observation may have been extended, with a collimated jet-like emission away from the accretion disk up to $\sim 26$ gravitational radii $\left(R_{\mathrm{g}}\right)$ above the back hole's rotational axis and confined to a small region within a few $R_{\mathrm{g}}$. They argue that particles were accelerated away from the accretion disk with the base of the unresolved jet possibly moving non-relativistically, and the particles would return under the gravitational effects of the central engine to still sufficiently illuminate the disk with a low $(<1.0)$ reflection fraction yield.

3. Even though we have sampled high counts per bin with $\chi^{2}$-statistics here, we can still be subject to bias in results because our total number of data bins to be fitted (973) is not lower than the squared-root of the total number of counts in the analyzed energy range (Humphrey et al. 2009).

4. The treatment of X-radiation from astrophysical black holes assumes the thin disk model is computationally and physically stable at large, but constitutes typical, oversimplifying assumptions on the disk conditions (Page \& Thorne 1974; Bambi 2017b). Taylor \& Reynolds (2018) used the lamppost geometry for relativistic reflection spectroscopy using a thick accretion disk. It may be likely that the disk thickens (geometrically) with increasing mass accretion rate. At Eddington luminosity $>10 \%$, the inner edge of the accretion disk may move further inward than the thin disk approximation where it is thought to be at the ISCO (Abramowicz \& Lasota 1980). Keek \& Ballantyne (2016) found the analyzed data set here to be on the high end of an accretion rate with an extended corona, which seems to agree with the coronal geometry proposed in Gallo et al. (2015) and Wilkins \& Gallo (2015). Work is currently underway to model and test the effects with a thick disk.

\section{Conclusions}

The main goal of this work is to test how well we can recover the Kerr metric if we introduce a deformation in the Kerr spacetime around the supermassive black hole of NLS1 Mrk 335, using observational data. We use the XSPEC model RELXILL_NK, which is an extension of the widely used relativistic reflection code RELXILL, to include parametrically deformed Kerr metrics. We use the Suzaku FI XIS data set, studied in a large survey paper by Walton et al. (2013).

We find differences from the work of Walton et al. (2013). A PLC+RDC model does not explain the data completely and partial covering is needed to account for absorption. We obtain decent constraints on $a_{*}, \alpha_{13}$, and $\alpha_{22}$ (shown in Figure 4). At first glance, the results indicate two sets of contours, the nearKerr contours including the Kerr solution and following the results from previous studies, and the far-Kerr contours excluding the Kerr solution and including extreme values of deformation parameters. A thorough investigation (see Appendix A) suggests that the far-Kerr contours are not robust and sometimes have unreasonable parameter values. Their appearance in the planes of the deformation parameters against $a_{*}$ and absence with respect to other physical parameters like $\log \xi$ and $i$ with repeated tests indicates there exist uncertainties (possibly data quality and systematics snarled together) currently beyond our control. This is one reason to also say that it is not possible to completely rule out the far-Kerr solutions because they exist in at least one two-dimensional parameter phase space. However, except for the spin-deformation contours, all other tests undoubtedly vote in favor of the near-Kerr solutions, and thus, our inference in Section 4. 
The data analyzed in this study was found to have a best-fit output $\sim 14 \%-15 \%$ relative strength of relativistic reflection compared to the total contributed by the PLC and the blurred reflector in the RDC. ${ }^{10}$ This is the lowest reflection data set of Mrk 335 from Table 1. The proof of this also comes from Wilkins \& Gallo (2015), who proposed a change in coronal geometry from the 2006 XMM-Newton state to the Suzaku state (later the same year), and has become rather extended, beaming more radiation away from the disk in the latter state. The current study, seen from a different perspective, tests for a lower limit using the method of constraining gravity with weak reflection data for Mrk 335. The bounds can only get better from here with a synergy between stronger reflection, better photon counts and appreciable, simultaneous high-energy coverage (see Section 3.2 for a recap on available data and states).

A precision test of gravity using X-ray reflection spectroscopy is a nascent field and every study opens new challenges. Mrk 335 is not an easy source to analyze. The model employed here is the first time RELXILL_NK is combined with a partial covering absorber. The analysis performed here had unique challenges, and the fact that we could obtain constraints comparable to other studies suggests that it is indeed possible to use this technique for testing theories of gravity even in complex scenarios, provided one is careful during the analysis and interpretation of results. The constraints presented in this work are not the strongest (compared to other works in the recent past) owing to the reflection data quality, and certainly do not display the limitations of the X-ray reflection spectroscopy method.

We extend our gratitude to the anonymous referees for suggesting helpful tips in order to improve the quality of the paper. K.C. thanks Michael Parker and Matteo Guainazzi for their helpful comments on the data analysis part. The work of K.C., D.A., A.B.A., and C.B. was supported by the Innovation Program of the Shanghai Municipal Education Commission, grant No. 2019-01-07-00-07-E00035, the National Natural Science Foundation of China (NSFC), grant No. U1531117, and Fudan University, grant No. IDH1512060. K.C. also acknowledges support from the China Scholarship Council (CSC), grant No. 2015GXYD34. S.N. acknowledges support from the Excellence Initiative at Eberhard-Karls Universität Tübingen. A.B.A. also acknowledges the support from the Shanghai Government Scholarship (SGS). C.B. and J.A.G. also acknowledge support from the Alexander von Humboldt Foundation.

\section{Appendix A \\ The Near-Kerr and Far-Kerr Contours}

We now discuss the near-Kerr and far-Kerr contours of Figure 4. We can see that both panels indicate the presence of multiple minima bound at $99 \%$ confidence. While the nearKerr contours remain consistent in shape and size when the stepping resolution (i.e., spacing between two values of a parameter in the grid selected) is changed, the far-Kerr contours change significantly with changing resolution. Moreover, often the fits landing in the far-Kerr region tend to get stuck, and fail to constrain the deformation parameter

\footnotetext{
10 This was determined with the CFLUX model on XSPEC in the $0.6-10 \mathrm{keV}$ band.
}

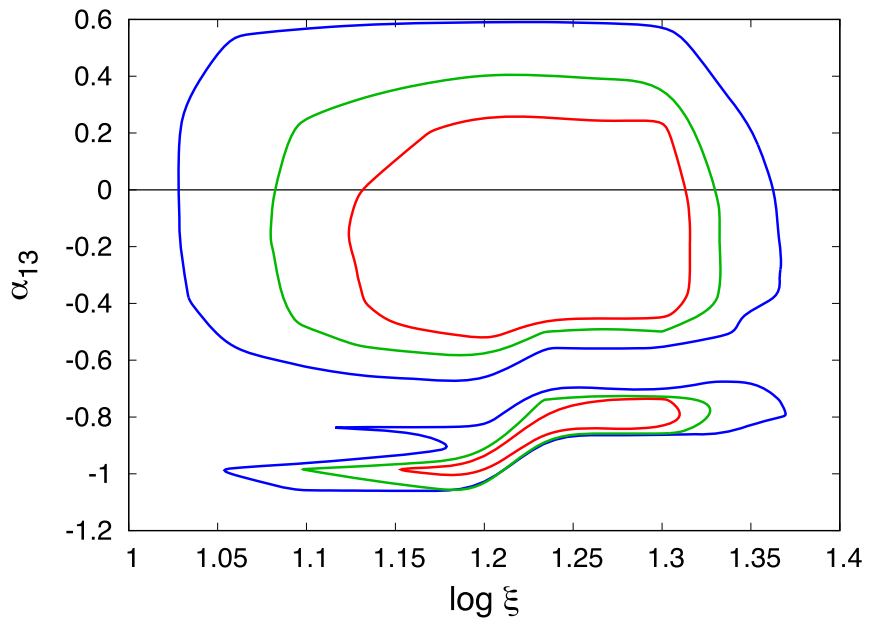

(a)

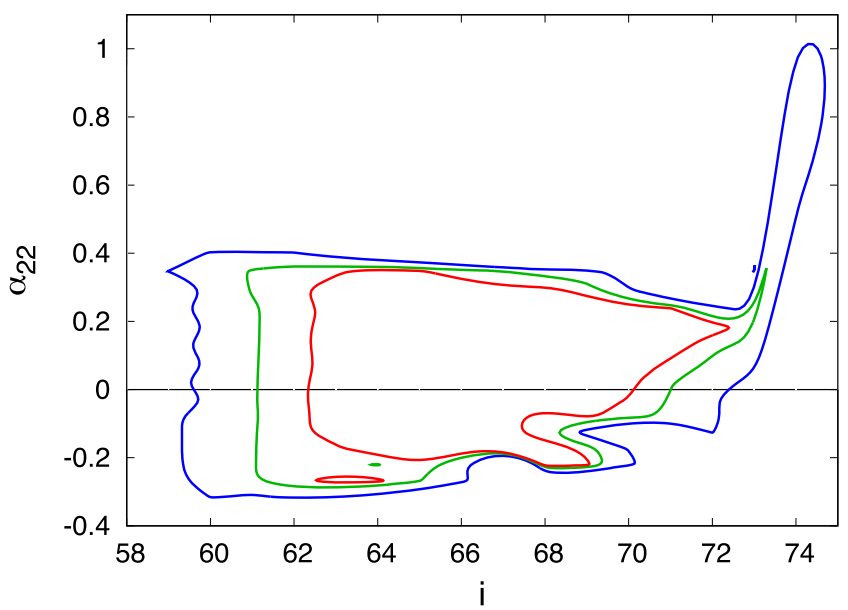

(b)

Figure 6. Panel (a): contour plot showing the degeneracy between $\alpha_{13}$ and $\log \xi$ assuming model D. Panel (b): contour plot showing the degeneracy between $\alpha_{22}$ and $i$ assuming model D. The red, green, and blue contour lines indicate $68 \%, 90 \%$, and $99 \%$ confidence levels, respectively.

at both upper and lower bounds. We emphasize at this point that the near-Kerr and far-Kerr solutions in each panel of Figure 4 are statistically similar (i.e., they have similar $\chi^{2} /$ d.o.f.). Even best-fit values of parameters (other than $a_{*}$, $\alpha_{13}$, and $\alpha_{22}$ ) show no systematic difference between fits in the two solution regions. Since the far-Kerr feature has never been observed in a similar analysis, we need to check if both near-Kerr and far-Kerr contours are physically reasonable and robust. Only statistical similarity is not sufficient to reach a conclusion when conducting sensitive tests, like with strong gravity, when a lot of parameters are involved in a complex modeling.

Apart from the fitting glitches (i.e., random fits getting stuck and the failure to yield bounds on the deformation parameters), some additional anomalous behavior were detected when fits would land in the far-Kerr minima. In the $\alpha_{13}$ case, for several stepping resolutions, a random fit in the far-Kerr minimum showed a drop in the disk temperature $(\log \xi<1.0)$. No other parameter was affected. To investigate further, we calculate the two-dimensional degeneracy between $\alpha_{13}$ and $\log \xi$. Figure 6(a) shows this contour. We 


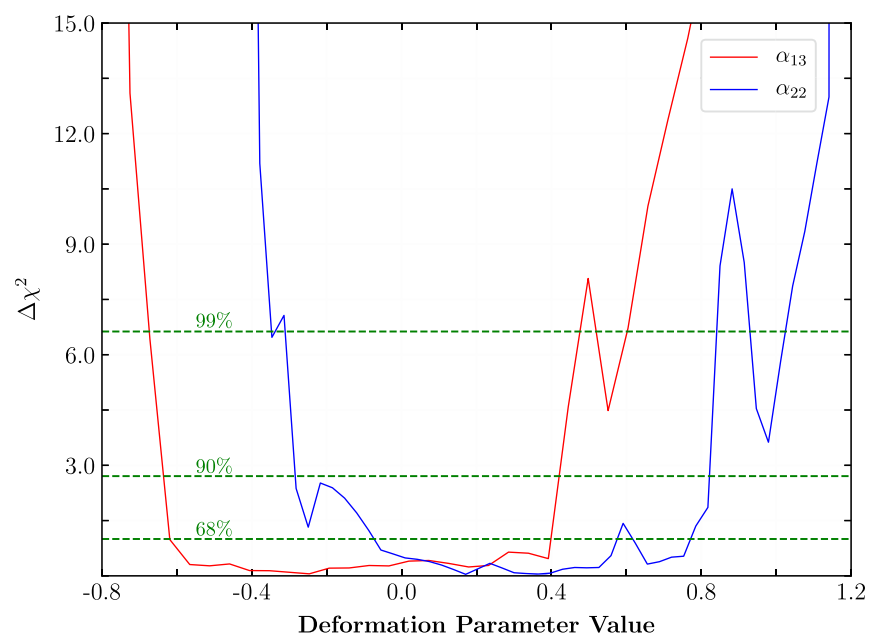

Figure 7. Plot shows the one-dimensional contours of the deformation parameters $\alpha_{13}$ and $\alpha_{22}$ against the delta-fit statistic $\left(\Delta \chi^{2}\right)$ with indications for uncertainties at 1 parameter of interest under $68 \%, 90 \%$, and $99 \%$ confidence intervals.

Table 4

Examples of the Limited Domains of Model Parameters for the Final Crossexamination of the far-Kerr Solutions

\begin{tabular}{|c|c|c|}
\hline Component & Parameter & Range(s) \\
\hline \multirow[t]{5}{*}{ ZPCFABS } & $n_{\mathrm{H}}$ & $1-1000$ \\
\hline & & $1-100$ \\
\hline & & $2-10$ \\
\hline & CvrFract & $0.0-0.8$ \\
\hline & & $0.1-0.5$ \\
\hline \multirow[t]{2}{*}{ ZPOWERLAW } & PhoIndex & $2.0-2.8$ \\
\hline & & $2.3-2.6$ \\
\hline \multirow[t]{13}{*}{ RELXILL_NK } & $q^{\mathrm{a}}$ & $4-10$ \\
\hline & & $5-10$ \\
\hline & & $6-10$ \\
\hline & & $1-10$ \\
\hline & $a_{*}$ & $0.7-0.998$ \\
\hline & $i$ & $30-75$ \\
\hline & & $55-75$ \\
\hline & $\log \xi$ & $0.0-3.0$ \\
\hline & & $0.0-2.0$ \\
\hline & & $1.0-2.0$ \\
\hline & & $1.0-4.7$ \\
\hline & $A_{\mathrm{Fe}}$ & $0.5-5.0$ \\
\hline & & $0.5-2.0$ \\
\hline
\end{tabular}

Notes. All combinations of these sampled ranges were inspected. Parameter units are defined in either Table 2 or 3.

a Although Walton et al. (2013) proposed limiting $q \geqslant 3$, we tested with flatter emissivities included and saw that they lead to contours similar to those of Figure 9.

obtain closed contours with $\log \xi>1.0$ with no hint of extension to lower values, suggesting that the far-Kerr minimum with $\log \xi<1.0$ are outliers. The uncertainty in $\alpha_{13}$ from this plot is much more consistent with the near-Kerr contour rather than the far-Kerr contour. In the case of $\alpha_{22}$, random far-Kerr fits showed a drop in the inclination values.
To investigate further, we compute the two-dimensional $\alpha_{22}-i$ degeneracy. This result is shown in Figure 6(b). Here again we obtain closed contours. The uncertainty in inclination from this plot matches well with that obtained from Figure 5 and the error in $\alpha_{22}$ is consistent with the nearKerr contour rather than the far-Kerr contour of Figure 4(b). This again suggests that the far-Kerr contours cover a disconnected parameter space and are spurious.

We performed an additional test to check for the robustness of the uncertainty in deformation parameters. Instead of a twodimensional STEPPAR which shows the degeneracy of a deformation parameter with another model parameter, we perform a one-dimensional STEPPAR with just the deformation parameter. This result is shown in Figure 7. Here we very clearly obtain a contour around the Kerr solution (corresponding to zero deformation parameter) and the range of deformation parameter is consistent with that obtained from the near-Kerr contours of Figure 4.

The solutions were further subjected to a final test, by limiting the grids of physical parameters to sensible ranges (Table 4) and checking the results for any difference. We discovered no startling change. The far-Kerr solutions do not disappear from Figure 4 nor do they appear in Figure $6 .{ }^{11}$ At this point we caution that we do not claim whether the far-Kerr solutions are physical or not, but simply describe what this lowreflection data set shows us.

\section{Appendix B Ionized versus Un-ionized Partial Covering}

When applying a partial covering absorber, it was not clear at the onset whether the absorber is ionized or un-ionized. We chose to model the ionized absorber with ZXIPCF and warm absorbers built on XSTAR (Bautista \& Kallman 2001). The unionized one was modeled with ZPCFABS. With ZXIPCF we did manage to get a better fit over the use of ZPCFABS, where the latter yielded the next-to-best statistical convergence among all model combinations tested with. However, no constraint was obtained for the hydrogen $(\mathrm{HI})$ column $n_{\mathrm{H}}$ of the ionized covering against the $\mathrm{X}$-ray continuum index $\Gamma$. Among all absorbers, only the ZPCFABS model provided good constraints on $n_{\mathrm{H}}$ (see Figure 8), which indicates that the model is statistically restrained. Moreover, it is ZPCFABS that produced decent constraints on both deformation parameters (Figure 4) with comparatively 1 less d.o.f. than ZXIPCF. Figure 9 shows the constraint obtained with Model D if ZXIPCF was used instead. The model completely excludes the Kerr solution. The fit statistic is exceptionally good in this case $\left(\Delta \chi^{2} /\right.$ d.o.f. $=$ $986.78 / 958 \sim 1.03)$. Naively, one may get mislead by such statistics. But we have seen in other instances, e.g., $\mathrm{Xu}$ et al. (2018), that some model degeneracies result in exceptional fit statistics at highly non-Kerr solutions (i.e., excluding the Kerr solution at very large $\sigma$ ), but are usually the result of incorrect modeling. Moreover, we do not obtain any closed contour for the $\alpha_{22} \neq 0$ case with ZXIPCF. We thus conclude in favor of ZPCFABS.

\footnotetext{
${ }^{11}$ However, $\log \xi$ and $i$ displayed lower uncertainties on the near-Kerr deformation parameter bounds, further defying the far-Kerr solutions.
} 


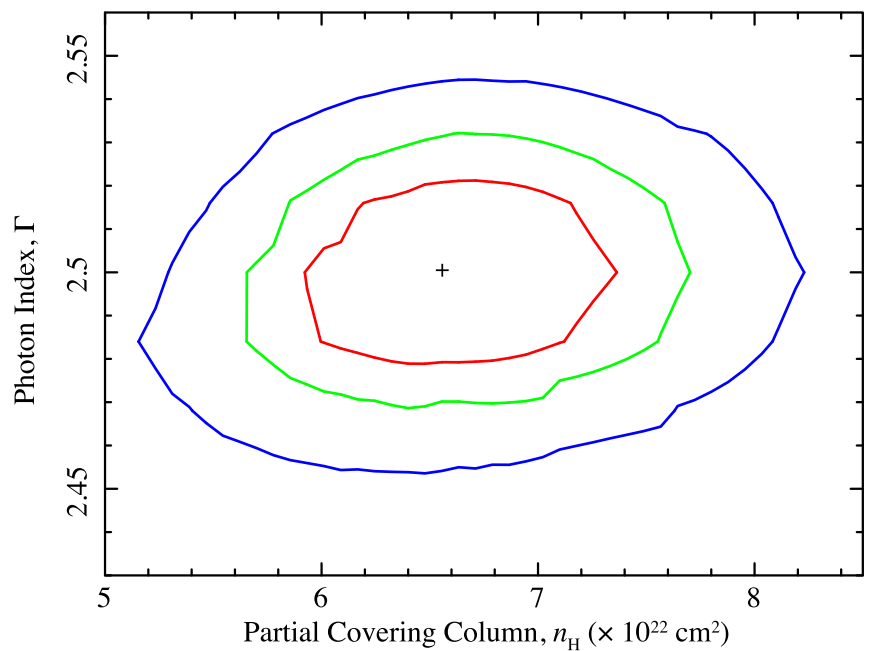

(a)

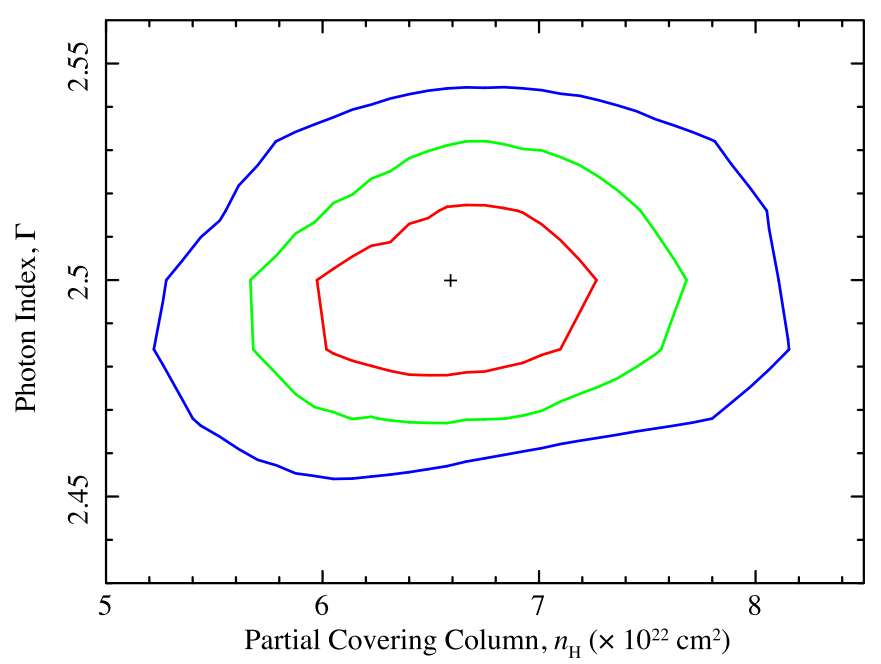

(b)

Figure 8. Two-dimensional contour plots showing the degeneracy between the spectral photon index parameter $\Gamma$ vs. the partial covering hydrogen column $n_{\mathrm{H}}$ in Model $\mathrm{D}$, for $\alpha_{13} \neq 0$ (left) and $\alpha_{22} \neq 0$ (right). Plot was rebinned on XSPEC for clarity.

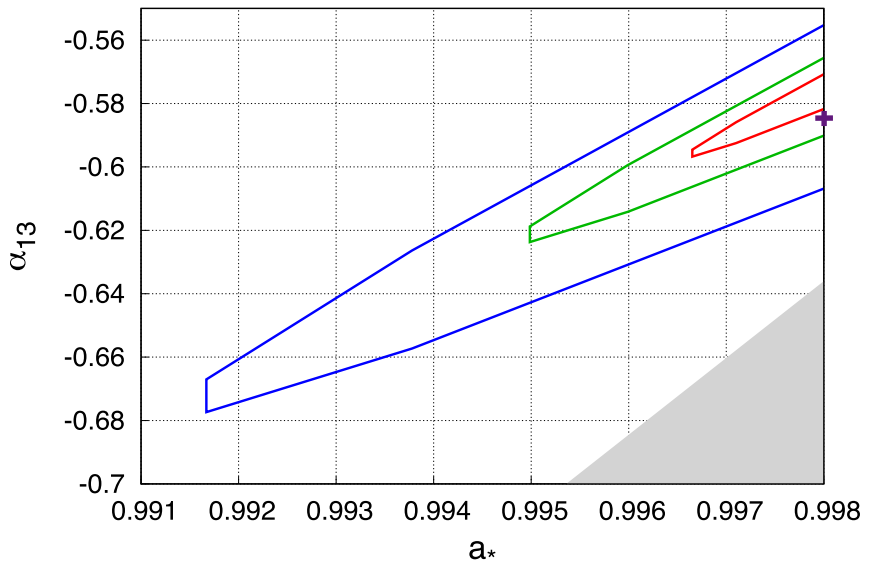

Figure 9. Similar to Figure 4(a), but using ZXIPCF instead of ZPCFABS. The purple "+" marks the best fit. Note that the Kerr solution $\left(\alpha_{13}=0\right)$ is not recovered.

\section{ORCID iDs}

Cosimo Bambi (ib https://orcid.org/0000-0002-3180-9502

\section{References}

Abramowicz, M. A., \& Lasota, J. P. 1980, AcA, 30, 35

Arnaud, K. A. 1996, in ASP Conf. Ser. 101, Astronomical Data Analysis Software and Systems V, ed. G. H. Jacoby \& J. Barnes (San Francisco, CA: ASP), 17

Ballantyne, D. R. 2017, MNRAS, 472, L60

Ballantyne, D. R., Iwasawa, K., \& Fabian, A. C. 2001, MNRAS, 323, 506

Bambi, C. 2013, PhRvD, 87, 023007

Bambi, C. 2017a, RvMP, 89, 025001

Bambi, C. 2017b, Black Holes: A Laboratory for Testing Strong Gravity (Singapore: Springer Nature)

Bambi, C., Dolgov, A. D., \& Petrov, A. A. 2009, JCAP, 9, 013

Bambi, C., Jiang, J., \& Steiner, J. F. 2016, CQGra, 33, 064001

Bambi, C., Malafarina, D., \& Tsukamoto, N. 2014, PhRvD, 89, 127302

Bambi, C., Modesto, L., \& Rachwał, L. 2017, JCAP, 1705, 003

Bambi, C., \& Nampalliwar, S. 2016, EL, 116, 30006

Bambi, C., Abdikamalov, A., Ayzenberg, D., et al. 2018, Univ, 4, 79

Bambi, C., Cárdenas-Avendaño, A., Dauser, T., García, J. A., \& Nampalliwar, S. 2017, ApJ, 842, 76

Bautista, M. A., \& Kallman, T. R. 2001, ApJS, 134, 139

Beheshtipour, B., Krawczynski, H., \& Malzac, J. 2017, ApJ, 850, 14

Berti, E., Barausse, E., Cardoso, V., et al. 2015, CQGra, 32, 243001

Brenneman, L. W., \& Reynolds, C. S. 2006, ApJ, 652, 1028

Buisson, D. J. K., Parker, M. L., Kara, E., et al. 2018, MNRAS, 480, 3689

Cao, Z., Nampalliwar, S., Bambi, C., Dauser, T., \& García, J. A. 2018, PhRvL, 120, 051101

Carter, B. 1971, PhRvL, 26, 331

Choudhury, K., García, J. A., Steiner, J. F., \& Bambi, C. 2017, ApJ, 851, 57

Crummy, J., Fabian, A. C., Gallo, L., \& Ross, R. R. 2006, MNRAS, 365, 1067

Dauser, T., García, J. A., Wilms, J., et al. 2013, MNRAS, 430, 1694

Dauser, T., Wilms, J., Reynolds, C. S., \& Brenneman, L. W. 2010, MNRAS, 409, 1534

Dvali, G., \& Gomez, C. 2013, ForPh, 61, 742

Fabian, A. C., Iwasawa, K., Reynolds, C. S., \& Young, A. J. 2000, PASP, 112,1145

Fabian, A. C., Rees, M. J., Stella, L., \& White, N. E. 1989, MNRAS, 238, 729

Gallo, L. C., Fabian, A. C., Grupe, D., et al. 2013, MNRAS, 428, 1191

Gallo, L. C., Gonzalez, A. G., Waddell, S. G. H., et al. 2019, MNRAS, 484, 4287

Gallo, L. C., Wilkins, D. R., Bonson, K., et al. 2015, MNRAS, 446, 633

García, J., Dauser, T., Lohfink, A., et al. 2014, ApJ, 782, 76

García, J., Dauser, T., Reynolds, C. S., et al. 2013, ApJ, 768, 146

García, J., \& Kallman, T. R. 2010, ApJ, 718, 695

George, I. M., \& Fabian, A. C. 1991, MNRAS, 249, 352

Giannuzzo, E. M., \& Stirpe, G. M. 1996, A\&A, 314, 419

Giddings, S. B. 2014, PhRvD, 90, 124033

Giddings, S. B. 2017, NatAs, 1, 0067

Gondoin, P., Orr, A., Lumb, D., \& Santos-Lleo, M. 2002, A\&A, 388, 74

Grier, C. J., Peterson, B. M., Pogge, R. W., et al. 2012, ApJL, 744, L4

Grupe, D., Komossa, S., \& Gallo, L. C. 2007, ApJL, 668, L111

Grupe, D., Komossa, S., Gallo, L. C., et al. 2008, ApJ, 681, 982

Grupe, D., Komossa, S., Gallo, L. C., et al. 2012, ApJS, 199, 28

Guilbert, P. W., \& Rees, M. J. 1988, MNRAS, 233, 475

Harrison, F. A., Craig, W. W., Christensen, F. E., et al. 2013, ApJ, 770, 103

Humphrey, P. J., Liu, W., \& Buote, D. A. 2009, ApJ, 693, 822

Jansen, F., Lumb, D., Altieri, B., et al. 2001, A\&A, 365, L1

Jiang, J., Bambi, C., \& Steiner, J. F. 2015a, JCAP, 5, 025

Jiang, J., Bambi, C., \& Steiner, J. F. 2015b, ApJ, 811, 130

Johannsen, T. 2013, PhRvD, 88, 044002

Johannsen, T. 2016, CQGra, 33, 124001

Johannsen, T., \& Psaltis, D. 2013, ApJ, 773, 57

Kalberla, P. M. W., Burton, W. B., Hartmann, D., et al. 2005, A\&A, 440, 775

Kara, E., Fabian, A. C., Cackett, E. M., et al. 2013, MNRAS, 434, 1129

Keek, L., \& Ballantyne, D. R. 2016, MNRAS, 456, 2722

Kerr, R. P. 1963, PhRvL, 11, 237

Konoplya, R., Rezzolla, L., \& Zhidenko, A. 2016, PhRvD, 93, 064015

Koyama, K., Tsunemi, H., Dotani, T., et al. 2007, PASJ, 59, 23

Laor, A. 1991, ApJ, 376, 90

Larsson, J., Miniutti, G., Fabian, A. C., et al. 2008, MNRAS, 384, 1316

Lightman, A. P., \& White, T. R. 1988, ApJ, 335, 57 
Longinotti, A. L., Krongold, Y., Kriss, G. A., et al. 2013, ApJ, 766, 104

Longinotti, A. L., Sim, S. A., Nandra, K., \& Cappi, M. 2007, MNRAS, 374,237

Mathur, S. D. 2005, ForPh, 53, 793

Miller, J. M. 2007, ARA\&A, 45, 441

Mitsuda, K., Bautz, M., Inoue, H., et al. 2007, PASJ, 59, S1

Nampalliwar, S., Bambi, C., Kokkotas, K. D., \& Konoplya, R. A. 2018, PhLB, 781,626

Nampalliwar, S., Xin, S., Srivastava, S., et al. 2019, arXiv:1903.12119

Nandra, K., George, I. M., Mushotzky, R. F., Turner, T. J., \& Yaqoob, T. 1997, ApJ, 477, 602

Ni, Y., Zhou, M., Cárdenas-Avendaño, A., et al. 2016, JCAP, 7, 049

Novikov, I. D., \& Thorne, K. S. 1973, in Black Holes (Les Astres Occlus), ed. C. Dewitt \& B. S. Dewitt (New York: Gordon and Breach), 343

O'Neill, P. M., Nandra, K., Cappi, M., Longinotti, A. L., \& Sim, S. A. 2007, MNRAS, 381, L94

Page, D. N., \& Thorne, K. S. 1974, ApJ, 191, 499

Parker, M. L., Wilkins, D. R., Fabian, A. C., et al. 2014, MNRAS, 443, 1723

Patrick, A. R., Reeves, J. N., Porquet, D., et al. 2011, MNRAS, 411, 2353

Price, R. H. 1972, PhRvD, 5, 2419

Rakshit, S., Stalin, C. S., Chand, H., \& Zhang, X.-G. 2017, ApJS, 229, 39

Reynolds, C. S., \& Fabian, A. C. 2008, ApJ, 675, 1048

Robinson, D. C. 1975, PhRvL, 34, 905

Ross, R. R., \& Fabian, A. C. 2005, MNRAS, 358, 211
Schee, J., \& Stuchlík, Z. 2009, GReGr, 41, 1795

Takahashi, T., Abe, K., Endo, M., et al. 2007, PASJ, 59, 35

Tananbaum, H., Peters, G., Forman, W., et al. 1978, ApJ, 223, 74

Taylor, C., \& Reynolds, C. S. 2018, ApJ, 855, 120

Tortosa, A., Bianchi, S., Marinucci, A., Matt, G., \& Petrucci, P. O. 2018, A\&A, 614, A37

Tripathi, A., Nampalliwar, S., Abdikamalov, A. B., et al. 2018, PhRvD, 98, 023018

Tripathi, A., Nampalliwar, S., Abdikamalov, A. B., et al. 2019a, ApJ, 875, 56

Tripathi, A., Yan, J., Yang, Y., et al. 2019b, ApJ, 874, 135

Walton, D. J., Nardini, E., Fabian, A. C., Gallo, L. C., \& Reis, R. C. 2013, MNRAS, 428, 2901

Wang-Ji, J., Abdikamalov, A. B., Ayzenberg, D., et al. 2018, arXiv:1806. 00126

Wilkins, D. R., \& Gallo, L. C. 2015, MNRAS, 449, 129

Will, C. M. 2014, LRR, 17, 4

Wilms, J., Allen, A., \& McCray, R. 2000, ApJ, 542, 914

Xu, Y., Nampalliwar, S., Abdikamalov, A. B., et al. 2018, ApJ, 865, 134

Yagi, K., \& Stein, L. C. 2016, CQGra, 33, 054001

Yunes, N., \& Siemens, X. 2013, LRR, 16, 9

Zhang, Y., Abdikamalov, A. B., Ayzenberg, D., et al. 2019, ApJ, 875, 41

Zhou, M., Cardenas-Avendano, A., Bambi, C., Kleihaus, B., \& Kunz, J. 2016, PhRvD, 94, 024036

Zhou, M., Cao, Z., Abdikamalov, A., et al. 2018, PhRvD, 98, 024007 